\title{
Biomasa microbiana y respiración basal del suelo bajo sistemas agroforestales con cultivos de café
}

\section{Microbial biomass and basal soil respiration under agroforestry systems with coffee crops}

\author{
Yuri J. Pardo-Plaza ${ }^{1}$; Jorge E. Paolini Gómez²; Miriam Elena Cantero-Guevara³
}

\begin{abstract}
1'Ing. Agrónomo, M.Sc. Universidad de Córdoba, Departamento de Química, Montería, Colombia, e-mail: yjpardoplaza@correo.unicordoba.edu.co, (Dhttps://orcid.org/0000-0002-5264-1606

Licenciado en Química, Dr. Sc. Agr. Instituto Venezolano de Investigaciones Científicas, Centro de Ecología, Caracas, Venezuela, e-mail: mapire3000@yahoo. com, (Dhttps://orcid.org/0000-0001-5644-1671

${ }^{3}$ Lic. Química y Biología, M.Sc. Departamento de Química. Universidad de Córdoba, Montería, Colombia, e-mail: cmelengca@gmail.com, Dhttps://orcid. org/0000-0001-8941-1276
\end{abstract}

Cómo citar: Pardo-Plaza, Y.J.; Paolini Gómez, J.E.; Cantero-Guevara, M.E. 2019. Biomasa microbiana y respiración basal del suelo bajo sistemas agroforestales con cultivos de café. Rev. U.D.C.A Act. \& Div. Cient. 22(1):e1144. https://doi.org/10.31910/rudca.v22. $\mathrm{n} 1.2019 .1144$

Artículo de acceso abierto publicado por Revista U.D.C.A Actualidad \& Divulgación Científica bajo una licencia Creative Commons CC BY-NC 4.0

Recibido: Diciembre 27 de 2017

Aceptado: Mayo 14 de 2019

\section{RESUMEN}

La actividad microbiológica es esencial para mantener la calidad de los suelos y los sistemas agroforestales surgen como alternativa, para el manejo agroecológico y sostenible del suelo. Este estudio evaluó el efecto de las variedades de café (Caturra y Catuaî) y de las fluctuaciones por épocas climáticas, sobre algunas propiedades microbiológicas del suelo, como indicadores de calidad, en un sistema agroforestal. Las muestras de suelo, se tomaron en la capa superior, a $5 \mathrm{~cm}$ de profundidad, durante un año en épocas seca y lluviosa. Las mayores emisiones de $\mathrm{CO}_{2}$, se observaron en los suelos con la variedad Catuaí, en época seca. Los niveles de carbono de la biomasa microbiana (Cmic) no mostraron diferencias entre las variables estudiadas. Los valores obtenidos para el cociente metabólico $\left(\mathrm{qCO}_{2}\right)$ fueron mayores en los suelos con la variedad Catuaí, mientras que el cociente microbiano (qMic) presentó los mayores valores en los suelos con la variedad Caturra. El cociente de eficiencia metabólica ( $\left.\mathrm{qCO}_{2} . \mathrm{Corg}^{-1}\right)$ no mostró diferencias entre los suelos estudiados; sin embargo, sus niveles fueron más altos para las muestras tomadas durante la época seca. La microbiota del suelo denotó sensibilidad a los cambios por época climática de muestreo y tipo de variedad cultivada, mientras que las constantes ecofisiológicas resultaron sensiblemente apropiadas, para evaluar la calidad del suelo.

Palabras clave: actividad microbiológica; suelo; café; agroecosistemas; constantes ecofisiológicas. Thesaurus: USDA https://agclass. nal.usda.gov/

\section{ABSTRACT}

The microbiological activity is essential to maintain soil quality, and agroforestry systems emerge as an alternative to the agro-ecological and sustainable land management. This work evaluated the effect of the Caturra and Catuaí coffee varieties, and the weather fluctuation on some microbiological properties of the soil, as indicators of quality in an agroforestry system. The soil samples were taken from the top layer with a depth of $5 \mathrm{~cm}$, during a year in dry and rainy seasons. The highest $\mathrm{CO}_{2}$ emissions were observed in soils with the Catuaí variety, in the dry season. On the other hand, Carbon levels 
of the microbial biomass (Cmic) did not show differences between the variables studied. The values obtained for the metabolic quotient $\left(\mathrm{qCO}_{2}\right)$ were higher in soils with the Catuai variety; while the microbial quotient (qMic) presented the highest values in soils with the Caturra variety. The metabolic efficiency ratio $\left(\mathrm{qCO}_{2} \cdot \mathrm{Corg}^{-1}\right)$ showed no differences between the studied soils, however, their levels were higher for the samples taken during the dry season. Soil microbiota showed sensitivity to changes by climatic period and by the type of variety, while the constant eco-physiological were substantially appropriate to evaluate soil quality and sensitive to changes by climatic period and variety of coffee grown in these agroecosystems.

Keywords: microbiological activity; soil; coffee; agroecosystem; ecophysiological constants. Thesaurus: USDA https://agclass.nal. usda.gov/

\section{INTRODUCCIÓN}

En Venezuela, al igual que en muchos países de Centro y Sur de América, el café tradicionalmente se cultiva bajo sombra de árboles del género Inga, Cordia, Cedrela y Erythrina, aunque también de árboles frutales, como aguacate (Persea americana), cítricos (Citrus sinensis) y bananos (Musa paradisiaca), conocido como sistema cafetalero multiestrato o policultivo tradicional, que mantiene la biodiversidad funcional y estructural del ecosistema (Perfecto \& Armbrecht, 2002). Estos árboles proveen de sombra al cultivo y mejora la calidad del café, reciclan nutrientes de las capas profundas del suelo, protegen al arbusto del café del viento, la lluvia y del exceso de sol, ayudan a regular la variabilidad climática, estabilizan el suelo al reducir la erosión y la densidad aparente, incrementan la infiltración del agua y proveen de nitrógeno al cultivo, a través de la fijación biológica del nitrógeno (Rapidel et al. 2015). Los sistemas agroforestales son considerados alternativas de uso sostenible del suelo y del ambiente, capaces de aumentar los niveles de productividad de los cultivos de pequeños agricultores, con la consecuente mejora de la calidad del suelo (Cardoso et al. 2005). Para evaluar la calidad del suelo, se debe considerar que los microorganismos son fundamentales en el proceso de descomposición de los residuos orgánicos del suelo e influyen sobre los ecosistemas y su fertilidad, interviniendo, tanto en el establecimiento de los ciclos biogeoquímicos como en la formación de la estructura del suelo (Hernández et al. 2003).

El carbono orgánico del suelo es uno de los más importantes indicadores de la calidad del suelo y está directamente relacionado con el mantenimiento de su estructura, infiltración, suministro y almacenamiento del agua, presencia de diferentes grupos de microorganismos, mineralización de la materia orgánica y la disponibilidad de nutrientes. La materia orgánica del suelo es una fuente de energía para los microorganismos, de disponibilidad y calidad de sustratos y la biodiversidad necesaria para mantener muchas de las funciones del suelo. Los indicadores biológicos, incluyen, propiedades asociadas con la actividad biológica sobre la materia orgánica, como la biomasa microbiana, la respiración basal y las actividades enzimáticas; además pueden incluir varios índices, como abundancia, diversidad, cadenas alimenticias, estabilidad de las comunidades de microorganismos, así como organismos asociados a la mesofauna, como las lombrices, anélidos, nematodos y artrópodos (Martínez-Salgado et al. 2010). La calidad del suelo de los bosques depende de numerosas variables físicas, químicas, biológicas y bioquímicas. Dentro de estas variables, las microbiológicas (biomasa microbiana y respiración basal) y las bioquímicas (actividades enzimáticas) son las más sensibles, por lo tanto, pueden proveer información de los cambios ocasionados por el estrés ambiental y las actividades antrópicas. Si se tiene en cuenta que la actividad microbiológica es esencial para mantener la calidad de los suelos, la biomasa microbiana y su actividad son aspectos que deben ser considerados para determinar el estado de un sistema o para entender cómo la actividad humana altera los ciclos biogeoquímicos, al comparar zonas naturales y degradadas (Bastida et al. 2008). Por otra parte, la comparación de los rendimientos de diferentes biomasas microbianas o el estudio de las influencias ambientales sobre cualquier función parece más útil, cuando la actividad es expresada en términos de constantes fisiológicas, como el cociente microbiano (qMic) y el cociente metabólico $\left(\mathrm{qCO}_{2}\right) \mathrm{o}$, en otras palabras, la aplicación de las constantes ecofisiológicas son otra forma de cuantificar los efectos de los cambios ambientales y antrópicos sobre las comunidades microbianas (Dilly, 2005).

El presente estudio tuvo como objetivo evaluar el efecto de dos variedades de café (Caturra y Catuai) y de las fluctuaciones por época climática (seca y lluvia) sobre las propiedades microbiológicas del suelo, como indicadores de calidad en un sistema agroforestal.

\section{MATERIALES Y MÉTODOS}

Área de estudio. Esta investigación, se desarrolló en la estación experimental Jaime Henao Jaramillo, de la Universidad Central de Venezuela (UCV), ubicada en la región de Baruta, Municipio Guaicaipuro, Estado Miranda, Venezuela, 10²2'24" N; 66 $54^{\circ} 04^{\prime \prime}$ W; 1.241 m.s.n.m. Presenta un ambiente montañoso de relieve accidentado, con un desnivel máximo de $342 \mathrm{~m}$ (cotas 1.130 - 1.472m) y pendiente promedio de $50 \%$. La temperatura media en la estación es $19,9^{\circ} \mathrm{C}$, humedad relativa de $83,8 \%$ y precipitación anual de $1321,5 \mathrm{~mm}$, siendo los meses húmedos de mayo a noviembre y los meses secos y fríos, diciembre a abril (Figura 1). Los suelos son franco-arcillosos, descritos, según Torres et al. (2009), como Humic Haplults, francosa fina, mixta, isohipertérmica, en la clasificación americana (USDA) y Haplic Acrisols, en la clasificación de la FAO.

La variedad Catuaí tenía una edad aproximada de 20 años y la variedad Caturra, se estableció, aproximadamente, hacía 8 años, en suelos que venían sembrados con la variedad Catuaí. La densidad de siembra fue de 6.410 y 4.200 plantas.ha $^{-1}$, para las variedades Caturra y Catuaí, respectivamente. La densidad de los árboles de sombra fue 44 árboles.ha ${ }^{-1}$, en promedio. El sitio experimental fue un agroecosistema de café bajo sombra de guamos (Inga spp.) y bucares (Erythrina poeppigiana), sin aplicación de insumos agrícolas, lo cual, corresponde a una fertilización orgánica pasiva, debido a que los únicos insumos recibidos por el suelo solo provienen del mantillo, formado por residuos de árboles de sombra y café.

En cada parcela, se realizó un muestreo no sistemático en zigzag, para un total de seis puntos y, en cada punto, se colectó una muestra 


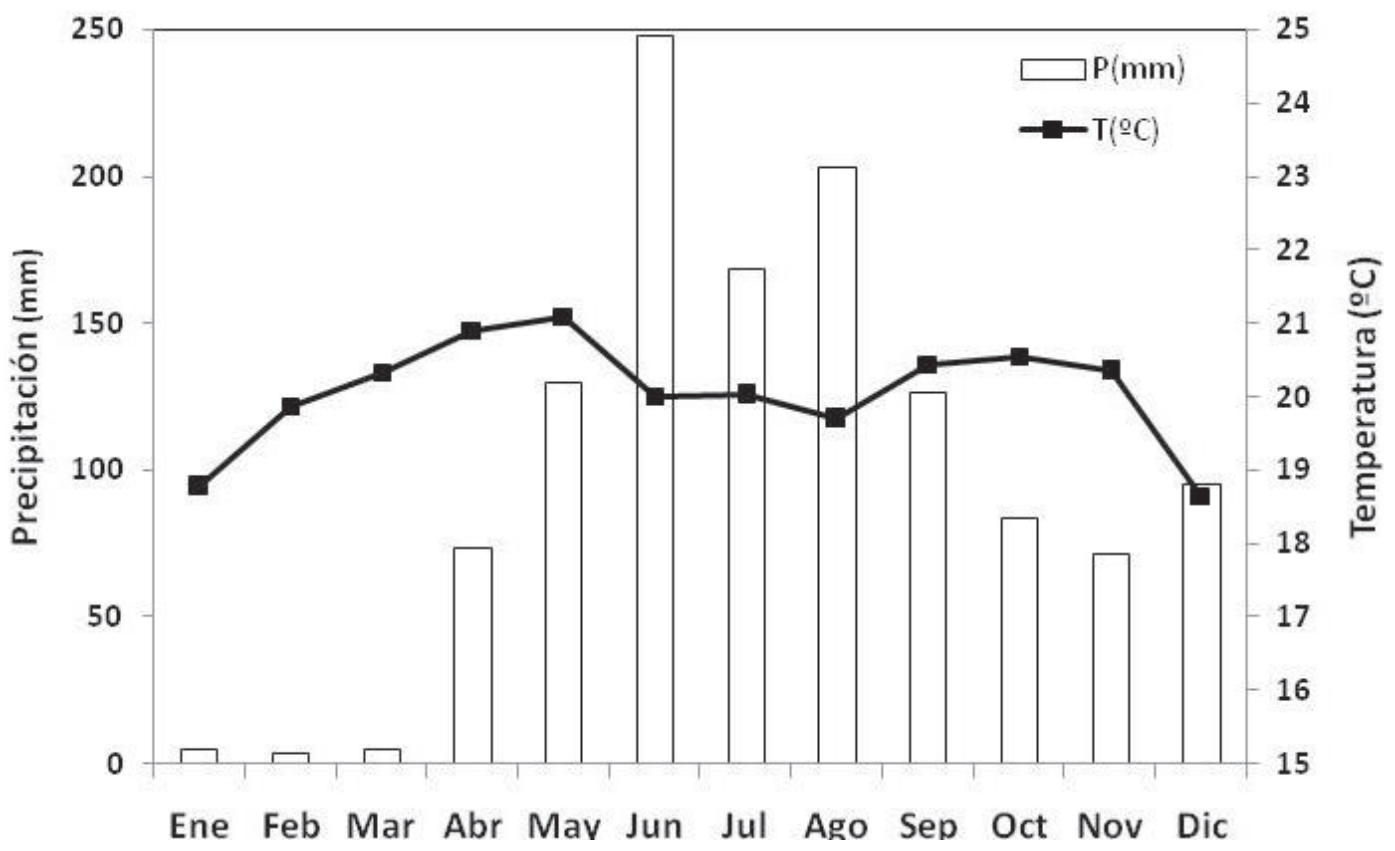

Figura 1. Variación de la precipitación promedio mensual y la temperatura promedio mensual en el Núcleo El Laurel, UCV.

superficial compuesta, a partir de seis-ocho muestras simples. Las mismas, se tomaron con un cilindro metálico de $10 \mathrm{~cm}$ de diámetro y $5 \mathrm{~cm}$ de altura entre los surcos. El muestreo, se realizó en época seca (enero, marzo y abril) y época de lluvia (junio, julio y septiembre).

Procedimientos analíticos. Los reactivos que se emplearon fueron grado analítico. Para los procedimientos analíticos, se siguieron los descritos por Paolini Gómez (2017). El Corg, se cuantificó espectrofotométricamente, después de la oxidación húmeda con dicromato de potasio y ácido sulfúrico concentrado y, posteriormente, se determinó el Cr (III) por espectrofotometría UV-Visible a $600 \mathrm{~nm}$ y se usó glucosa, como sustancia patrón (Heanes, 1984); la respiración basal (RB), se determinó por el método de adsorción en álcali (Pell et al. 2006). Se usaron $20 \mathrm{~g}$ de suelo fresco, llevados al $60 \%$ de su capacidad de retención de agua y se incubaron a $22 \pm 2{ }^{\circ} \mathrm{C}$, durante 7 días, con un vial lleno con $10 \mathrm{~mL}$ de hidróxido de sodio $0,3 \mathrm{M}$. La biomasa microbiana del suelo (Cmic), se midió usando el método de la respiración inducida por sustrato (RIS) (Höper, 2006). Se usaron los mismos suelos del experimento de respiración basal, a los cuales, se les adicionó $1 \mathrm{~mL}$ de una solución de glucosa al 8\% y se incubaron a $22 \pm 2^{\circ} \mathrm{C}$, con $10 \mathrm{~mL}$ de hidróxido de sodio $0,05 \mathrm{M}$, durante 6 horas. $\mathrm{El} \mathrm{CO}_{2}$ liberado por el consumo de la glucosa es atrapado por $\mathrm{NaOH}$; luego, se precipitó como $\mathrm{BaCO}_{3}$ con $2 \mathrm{~mL}$ de $\mathrm{BaCl}_{2} 0,5 \mathrm{M}$ y el $\mathrm{NaOH}$ en exceso, se tituló con $\mathrm{HCl}$ patrón $0,1 \mathrm{M}$, usando fenolftaleína, como indicador. Para el cálculo de la biomasa microbiana, se usó el factor de conversión propuesto por Beck et

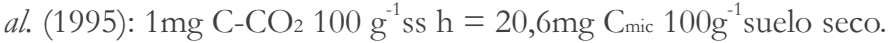

El cociente metabólico (qCO2), según Anderson \& Domsch (1993), se calculó como la relación entre $\mathrm{C}-\mathrm{CO}_{2} . \mathrm{C}_{\text {mic }}{ }^{-1}$; el cociente microbiano (qMic), como la relación entre $\mathrm{C}_{\text {mic. }} \mathrm{Corg}^{-1}$ (Sparling, 1992), mientras que el cociente de eficiencia metabólica, se definió como la relación qCO2. $\operatorname{Corg}^{-1}$ (Dilly, 2005).

Análisis estadístico. Los efectos de las variedades, la estacionalidad y su interacción sobre la respiración basal (RB), biomasa microbiana $\left(\mathrm{C}_{\mathrm{mic}}\right.$ ), las variables ecofisiológicas (qMic, $\mathrm{qCO}_{2}$ y qCO 2 . $\mathrm{Corg}^{-1}$ ) y carbono orgánico (Corg) del suelo, se analizaron mediante PERMANOVA (Permutational multivariate analysis of variance) de dos vías, previa comprobación del supuesto de homocedasticidad de dispersión multivariada mediante PERMDISP (Permutation analysis of multivariate dispersions); esta última prueba es similar al test de Levene (Anderson et al. 2008). Para todos los análisis, se normalizaron previamente los datos y se calcularon matrices de distancias euclidianas. Se utilizó el programa PRIMER 6. Versión 6.1.13 \& PERMANOVA + 1.0.3, para la realización de estos análisis.

\section{RESULTADOS Y DISCUSIÓN}

Estudios han demostrado que los suelos cultivados con café a gran altura bajo la sombra de árboles frutales o especies forestales producen altas adiciones de materia orgánica al suelo, lo que hace que sea una práctica importante para mantener altos niveles de carbono, mejorando la fertilidad y la actividad microbiana del suelo (Notaro et al. 2014). 
Carbono Orgánico (Corg). En la tabla 1, se relaciona los resultados obtenidos para las propiedades microbiológicas estudiadas, el Corg y los índices ecofisiológicos. El Corg mostró los mayores valores en suelos con la variedad Catuaí, en ambas épocas climáticas. Estos hallazgos, probablemente, se deben a que las plantas de esta variedad presentan mayor crecimiento vegetativo y mayor edad de establecimiento (aproximadamente, 20 años), con respecto a la variedad Caturra, cuyas plantas son más pequeñas y se estableció, aproximadamente, hacía 8 años, en suelos que se venían cultivando con la variedad Catuaí, por lo que se evidenció mayor perturbación en los suelos cultivados con la variedad Caturra. No hay diferencias significativas por la época climática. Los valores obtenidos para Corg son consistentes con otros estudios realizados en suelos, bajo agroecosistemas cafetaleros (Durango et al. 2015), en Ultisoles, con características ándicas de Turrialba - Costa Rica, encontrando mayores valores de Corg en suelos de bosque $\left(70 \mathrm{gC} \cdot \mathrm{kg}^{-1}\right)$, comparado con suelos cafetaleros $\left(57 \mathrm{gC} \cdot \mathrm{kg}^{-1}\right)$ y suelos con asociación café banano (39gC. $\mathrm{kg}^{-1}$ ) y Notaro et al. (2014), en la región semiárida de Pernambuco - Brasil, reportaron valores entre 21 y $45,6 \mathrm{gC} . \mathrm{kg}^{-1}$.

En otra investigación (Paolini Gómez, 2017) reportó resultados similares en Venezuela, en suelos cafetaleros, bajo manejo tradicional (agroforestal sin insumos) y cafetales a plena exposición solar, donde encontró valores de 35,0 y 29,0gC. $\mathrm{kg}^{-1}$, respectivamente, pero inferiores para cafetales, bajo manejo orgánico de $66,6 \mathrm{gC} \mathrm{kg}^{-1}$; este último valor supera a los del presente estudio. Otros trabajos reportan un valor mayor a los hallados en esta investigación, (Silva-Parra et al. 2017), en cafetales bajo manejo orgánico sobre Oxisoles y Ultisoles, en Villavicencio (Colombia), que indica un valor promedio de 53,1 $\mathrm{gC} \mathrm{kg}^{-1}$ y Pabst et al. (2013), en un estudio realizado en el Monte Kilimanjaro, en la región noreste de Tanzania, hallaron $18,9 \mathrm{gC} \cdot \mathrm{kg}^{-1}$, en plantaciones de café. Estos últimos autores, además observaron pérdidas del 10\% del Corg, debido al cambio en el uso del suelo de pastizales a plantaciones de café, posiblemente, por el uso intensivo de plaguicidas en la producción de café y a la remoción de los restos vegetales, después de la poda de los arbustos de café.
Por otra parte, estudios han demostrado que la principal fuente de nitrógeno en los suelos es el contenido de Corg y de materia orgánica (Sadeghian \& González, 2012), lo cual, puede estar relacionado con los valores de nitrógeno, observados es los suelos con la variedad Catuaí, en época lluviosa $\left(6,1 \pm 0,1 \mathrm{NT}\right.$ (gN.kg $\left.{ }^{-1}\right)$. De manera análoga, la capacidad de intercambio catiónico real evaluada en este estudio, registró variación altamente significativa $p<0,001$, entre variedades y por la época de muestreo, los valores fueron mayores en los suelos con la variedad Catuaí, en ambas épocas climáticas, siendo más altos en la estación lluviosa $\left(45,4 \pm 1,7 \mathrm{cmol}^{+} . \mathrm{kg}\right)$, que también se puede explicar por el mayor contenido de Corg, en estos suelos.

Respiración basal (RB). Se demostró que la actividad microbiana (respiración basal) muestra variación significativa, tanto por el tipo de variedad de café como por la época climática de muestreo. Los mayores valores, se observaron en la estación seca respecto a la lluviosa, para ambas variedades. Así, los valores que se obtuvieron, para la misma, fueron el $14 \%$ mayores en los suelos con la variedad Catuaí, estación seca, alcanzando $37,0 \pm 2,1 \mathrm{mgC}-\mathrm{CO}_{2} \mathrm{~kg}^{-1} \mathrm{~d}^{-1}$, comparado con $31,8 \pm 2,4 \mathrm{mgC}-\mathrm{CO}_{2} \mathrm{~kg}^{-1} \mathrm{~d}^{-1}$ en suelos con la variedad Caturra, estación seca; igualmente, los valores encontrados en suelos con la variedad Catuaí, estación lluviosa, fueron $26 \%$ superiores a los obtenidos en los suelos con la variedad Caturra, estación lluviosa (Tabla 1).

Los mayores valores hallados en la estación seca pueden estar relacionados con factores de estrés hídrico, debido a la reducción del contenido de agua del suelo o, probablemente, a un aumento de la actividad metabólica de la microbiota y biológica en general, favorecido por mejores condiciones de aireación del suelo. Por otra parte, las mayores pérdidas de $\mathrm{CO}_{2}$ encontradas en suelos con la variedad Catuaí estarían asociadas a mayores contenidos de Corg, observados en esos suelos; así, un mayor suministro de MOS podría favorecer mayor población de bacterias, que atacan rápidamente los sustratos orgánicos y aceleran los procesos de oxidación biológica (Notaro et al. 2014). Estos resultados reflejan que la actividad microbiana

Tabla. 1. Media \pm error estándar para las propiedades microbiológicas, constantes ecofisiológicas y Corg. Nivel de significancia para cada factor en PERMANOVA (Pman) y PERMDISP (pdisp) de dos vías $(* \mathrm{P}<0,05 ; * * \mathrm{P}<0,01 ; * * * \mathrm{P}<0,001$; ns $=$ no significativo al 95\%).

\begin{tabular}{|c|c|c|c|c|c|c|c|c|c|}
\hline \multirow{2}{*}{ Variable } & \multicolumn{2}{|c|}{ Catuaí } & \multirow{2}{*}{$\begin{array}{c}\text { Caturra } \\
\text { Seca }\end{array}$} & \multirow{2}{*}{$\begin{array}{c}\text { Caturra } \\
\text { lluvia }\end{array}$} & \multicolumn{2}{|c|}{ Variedad } & \multicolumn{2}{|c|}{ Estación } & \multirow{2}{*}{$\begin{array}{c}\text { Interacción } \\
\text { Pman }\end{array}$} \\
\hline & seca & Lluvia & & & Pman & pdisp & Pman & pdisp & \\
\hline $\mathrm{RB}$ & $37,0 \pm 2,1$ & $25,4 \pm 2,4$ & $31,8 \pm 2,7$ & $18,8 \pm 1,5$ & $* *$ & ns & $* * *$ & ns & ns \\
\hline Cmic & $340 \pm 25$ & $338 \pm 20$ & $363 \pm 18$ & $359 \pm 15$ & $\mathrm{~ns}$ & $\mathrm{~ns}$ & $\mathrm{~ns}$ & ns & $\mathrm{ns}$ \\
\hline qMic & $0,59 \pm 0,04$ & $0,59 \pm 0,04$ & $0,83 \pm 0,04$ & $0,88 \pm 0,05$ & $* * *$ & ns & ns & ns & ns \\
\hline $\mathrm{qCO}_{2}$ & $4,8 \pm 0,3$ & $3,2 \pm 0,3$ & $3,8 \pm 0,4$ & $2,2 \pm 0,2$ & ** & $\mathrm{ns}$ & $* * *$ & ns & $\mathrm{ns}$ \\
\hline $\mathrm{qCO}_{2 .} \operatorname{Corg}^{-1}$ & $83,7 \pm 7,2$ & $53,9 \pm 3,4$ & $88,8 \pm 11,4$ & $53,5 \pm 4,7$ & $\mathrm{~ns}$ & ns & $* * *$ & ns & $*$ \\
\hline Corg & $58,7 \pm 1,9$ & $58,3 \pm 2,5$ & $44,3 \pm 2$ & $42,09 \pm 2$ & $* * *$ & $\mathrm{~ns}$ & ns & $\mathrm{ns}$ & ns \\
\hline
\end{tabular}

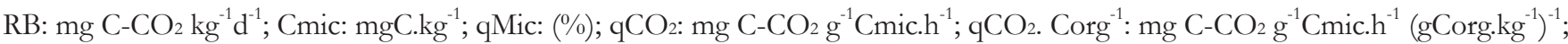
Corg: $\mathrm{gC} \cdot \mathrm{kg}^{-1}$ 
resultó sensible a las variaciones, tanto por el tipo de variedad como por la estacionalidad en los suelos cafetaleros, objeto de este estudio.

Los resultados obtenidos para la RB en este estudio superan los valores reportados en otros trabajos realizados en suelos, bajo diferentes sistemas con café; así, Paolini Gómez et al. (2008) observaron valores en el intervalo comprendido entre 15,5 y $26,0 \mathrm{mgC}^{-\mathrm{CO}_{2}} \mathrm{~kg}^{-1} \mathrm{~d}^{-1}$, en suelos cafetaleros, bajo diferente tipos de uso de la tierra (TUT), todos sistemas agroforestales, pero bajo manejo tradicional, en el estado Trujillo (Venezuela), resultados que pueden estar relacionados con la fuerte acidez de los suelos ( $\mathrm{pH}$ entre 4,3 y 4,6; contenido de aluminio intercambiable entre 2,3 y 4,8 cmol. $\mathrm{kg}^{-1}$ ). Recientemente, Paolini Gómez (2017), en sistemas agroforestales tradicionales y convencional de los Andes venezolanos, reportó valores de 55,1 y $51,2 \mathrm{mgC}-\mathrm{CO}_{2} \mathrm{~kg}^{-1} \mathrm{~d}^{-1}$, respectivamente, pero para cafetales bajo sistema agroforestal y manejo orgánico, encontró un valor promedio de $76,2 \mathrm{mgC}-\mathrm{CO}_{2} \mathrm{~kg}^{-1} \mathrm{~d}-1$.

Azevedo Júnior et al. (2017), en el estado de Espíritu Santo (Brasil), indican también altas tasas de respiración basal en cafetales bajo manejo orgánico de 70 y $60 \mathrm{mgC}-\mathrm{CO}_{2} \mathrm{~kg}^{-1} \mathrm{~d}^{-1}$, para las épocas de invierno (época seca) y verano (época de lluvia), respectivamente. Estos últimos autores detectan diferencias significativas entre las épocas de muestreo. Esta gran variedad de resultados reseñados en la literatura está relacionada con los factores que controlan la mineralización del carbono, como intrínsecos al suelo (textura, $\mathrm{pH}$, calidad y cantidad de la materia orgánica, contenidos de nutrientes, capacidad de intercambio catiónico, salinidad, presencia de metales pesados, tipo de suelo, entre otros), como climáticos (temperatura y precipitación), además de la forma de manejo del suelo (incorporación de residuos, fertilización, pesticidas) (Singer \& Munns, 2006).

Biomasa microbiana (Cmic). Esta variable no mostró variaciones significativas por época climática ni por la variedad durante el estudio (Tabla 1). Se puede observar una ligera tendencia a mayores valores en los suelos, bajo la variedad Caturra y en la estación seca (Tabla 1). El hecho de no detectar diferencias significativas de la biomasa microbiana para las épocas de muestreo y entre las variedades, tal vez se debe a que, sobre la superficie de los suelos cafetaleros bajo sombra, sin aplicación de insumos agrícolas y bajo fertilización orgánica pasiva, se forma un mantillo estable, a partir de la hojarasca y residuos vegetales, provenientes tanto de los arbustos de café como de los árboles de sombra.

Este mantillo de residuos orgánicos regula la dinámica del agua en el agroecosistema y permite un aporte permanente de $\mathrm{C}$ y sustancias orgánicas al suelo, usadas como fuentes de nutrientes y de energía por los microorganismos del suelo (Balota \& Chaves, 2011), favoreciendo el crecimiento y el desarrollo de las comunidades microbianas. La vegetación y el tipo de suelo son factores claves, que pueden modificar las características del suelo y son fundamentales para mantener una microbiota estable (Bastida et al. 2008).

Nuestros hallazgos están en concordancia con los reportados por Glaeser et al. (2010), quienes en suelos arcillo arenosos de Brasil, bajo diferentes sistemas agroforestales orgánicos y tomando mues- tras en dos épocas climáticas diferentes, no observaron diferencias significativas por épocas climáticas de muestreo, ni por los sistemas de manejo orgánico, en cultivos de café. Varios investigadores han encontrado en suelos cafetaleros valores de Cmic menores, bajo sistemas de manejo convencional, comparados con sistemas de manejo orgánico (Azevedo Júnior et al. 2017; Paolini Gómez, 2017) o con sistemas agroforestales de café (Abera \& Wolde-Meskel, 2013), mientras que, en otros estudios, se ha reportado mayores valores de Cmic, en el bosque nativo, comparado con diversos sistemas de manejo con café (Glaeser et al. 2010; Notaro et al. 2014; Lammel et al. 2015; Durango et al. 2015).

Cociente metabólico $\left(\mathrm{qCO}_{2}\right)\left(\mathrm{RB}^{\mathrm{C}} \mathrm{Cmic}^{-1}\right)$. Se calcula a partir de la respiración basal y la biomasa microbiana del carbono y es una medida de la eficiencia en el uso del carbono por los microorganismos; valores bajos de este parámetro indican un uso más eficiente del recurso (Anderson \& Domsch, 1993). Los mayores valores, se hallaron durante la estación seca, en suelos con la variedad Catuaí 4,8 $\pm 0,3 \mathrm{mgC}-\mathrm{CO}_{2} \mathrm{~g}^{-1} \mathrm{Cmich}^{-1}$, en comparación con los obtenidos en suelos con la variedad Caturra, en la misma época climática $3,8 \pm 0,4 \mathrm{mgC}-\mathrm{CO}_{2} \mathrm{~g}^{-1} \mathrm{Cmic}^{-1}{ }^{-1}$, mientras que, en la época lluviosa, en suelos con la variedad Catuaí, se obtuvo $3,2 \pm 0,3 \mathrm{mgC}-\mathrm{CO}_{2} \mathrm{~g}^{-1} \mathrm{Cmic}^{-1}$ y con la variedad Caturra, 2,2 $\pm 0,2 \mathrm{mgC}-\mathrm{CO}_{2} \mathrm{~g}^{-1} \mathrm{Cmich}^{-1}$ (Tabla 1).

La disminución en el contenido de humedad durante la estación seca causa estrés sobre los microorganismos, con el concomitante aumento de la respiración basal, lo cual, está de acuerdo con lo reportado por varios autores (Anderson \& Domsch, 1993; Dilly, 2005), quienes afirman que, mayores valores de $\mathrm{qCO}_{2}$ podrían reflejar un mayor requerimiento de la energía de mantenimiento de la comunidad microbiana de ese suelo, en otras palabras, los microorganismos deben catabolizar más carbono a $\mathrm{CO}_{2} \mathrm{e}$ incorporar menos sustratos a la biomasa microbiana.

Otro factor de estrés en los suelos sembrados con Catuaí, que puede causar un incremento en los valores de $\mathrm{qCO}_{2}$ es el $\mathrm{pH}$, el cual, fue significativamente menor en estos suelos (5,1 - 4,6, época seca y lluviosa, respectivamente), comparados con los valores de $\mathrm{pH}$ obtenidos para suelos con la variedad Caturra (5,8 - 5,4, época seca y lluviosa, respectivamente), que está de acuerdo con lo observado por Anderson \& Domsch (1993). Los valores del qCO2 observados en los suelos con la variedad Catuaí, también podrían estar relacionados con los valores superiores de actividad microbiana que, a su vez, pudo ser promovida por los mayores contenidos de Corg en los suelos, con la variedad Catuaí (Tabla 1). Estos resultados, se podrían explicar, teniendo en cuenta que los valores de $\mathrm{qCO}_{2}$ pueden aumentar cuando la MOS contiene altos niveles de compuestos orgánicos, fácilmente disponibles (Paolini Gómez, 2017).

El intervalo de valores de $\mathrm{qCO}_{2}$ hallado en este estudio, se considera como bajo, lo cual, indica la madurez de ambos ecosistemas, demostrada en otros estudios (Anderson \& Domsch, 1993; Dilly, 2005; Abera \& Wolde-Meskel, 2013). Por otra parte, los menores valores del $\mathrm{qCO}_{2}$ en los suelos con la variedad Caturra, sugieren que esta variedad puede inducir un uso más eficiente del $\mathrm{C}$, por la comunidad microbiana. Esto es importante, puesto que menor $\mathrm{C}$ del suelo será 
liberado como $\mathrm{CO}_{2}$ y mayor $\mathrm{C}$ del suelo será almacenado como $\mathrm{Cmic}$ (Dilly, 2005). Es de notar que otros estudios han demostrado que mayores valores de $\mathrm{qCO}_{2}$ se han observado en sistemas convencionales, cuando se comparan con sistemas orgánicos o agroforestales con café, en diferentes regiones del mundo: Abera \& Wolde-Meskel (2013), en Etiopía y Paolini Gómez (2017), en Venezuela.

Cociente Microbiano (qMic). La relación $\mathrm{Cmic} \mathrm{Corg}^{-1}$, también denominada "Cociente microbiano", se ha considerado como un buen indicador de las alteraciones en los procesos del suelo; valores mayores o menores del qMic, podrían expresar la acumulación o pérdida de C del suelo (Anderson et al. 1989). Los mayores valores para qMic, se obtuvieron en suelos con la variedad Caturra, en la época seca $0,88 \%$ y $0,83 \%$, en la época lluviosa, mientras que los menores valores, se observaron en suelos con la variedad Catuaí, $0,59 \%$, en ambas épocas climáticas. Estos valores para qMic son menores a los reportados en la literatura, resultados que, posiblemente, se deben a los altos valores de los contenidos de Corg, que variaron entre 42,1 y $58,7 \mathrm{gC}^{-\mathrm{kg}^{-1}}$ (Tabla 1 ).

Por otra parte, no se encontró diferencias significativas para los valores del $\mathrm{Cmic}$, entre las variedades, ni por época climática; sin embargo, se observa la tendencia de los valores más altos del Cmic, en los suelos con la variedad Caturra (Tabla 1), mientras que los valores obtenidos para el Corg son significativamente mayores en los suelos con la variedad Catuaí (Tabla 1), por lo tanto, el qMic es mayor en los suelos con la variedad Caturra (Tabla 1). Estos hallazgos nos indican que las comunidades microbianas en los suelos con la variedad Caturra son más eficientes en fijar mayor proporción de $\mathrm{C}$ entrante en $\mathrm{Cmic}$, como ha sido discutido por varios autores (Dilly, 2005; Balota \& Chaves, 2011).

El qMic integra las propiedades del suelo con relación al grado de colonización microbiana. Altos valores del qMic indica que el biotipo favorece el metabolismo energético y el establecimiento de muchos microorganismos (Dilly, 2005); en otros términos, significa mejor calidad del suelo y mayor eficiencia de las comunidades microbianas en el uso del C, de la materia orgánica del suelo.

Cociente de Eficiencia Metabólica ( $q \mathrm{CO}_{2} \cdot \operatorname{Corg}^{-1}$ ). Los menores valores, se observaron durante la época lluviosa para ambos suelos, con la variedad Catuaí, 53,9 y con Caturra, 53,4 [(mgC-CO 2 $\left.\left.\mathrm{g}^{-1} \mathrm{Cmic}^{-1}\right) \cdot\left(\mathrm{gCorg}^{-1} \mathrm{~g}^{-1} \mathrm{ss}\right)^{-1}\right]$, mientras que en la época seca, se obtuvo 83,7 y 88,8 [(mgC-CO $\left.\mathrm{g} \mathrm{g}^{-1} \mathrm{Cmic}^{-1}\right)\left(\mathrm{gCorgg}^{-1} \mathrm{ss}^{-1}\right]$, con Catuaí y Caturra, respectivamente (Tabla 1), lo que indica un uso más eficiente del carbono en la época lluviosa y, a su vez, un estado eco-fisiológico más estable (Dilly, 2005). Los resultados obtenidos permiten inferir que la microbiota de los suelos estudiados es metabólicamente más eficiente en el uso de los sustratos energéticos durante la estación lluviosa, favorecida por el contenido de humedad del suelo (menor estrés por sequía).

Es de notar que las propiedades microbiológicas del suelo, especialmente aquellas relacionadas con el flujo de energía y el reciclaje de nutrientes, responden de forma rápida y sensible a los cambios de las condiciones del suelo mucho antes que, por ejemplo, el carbono orgánico y, de este modo, suministran una información anticipada sobre las alteraciones de la calidad del mismo. Por otra parte, las constantes ecofisiológicas permiten integrar los componentes abióticos y bióticos y definen cada sistema, como son: el cociente microbiano (qMic), el cociente metabólico $\left(\mathrm{qCO}_{2}\right)$ y el cociente de eficiencia metabólica ( $\left.\mathrm{qCO}_{2} \cdot \mathrm{Corg}^{-1}\right)$.

Con base en los resultados obtenidos, se puede concluir que la microbiota del suelo mostró sensibilidad a los cambios por estacionalidad climática y, por el tipo de variedad, en los agroecosistemas con café establecidos en la Estación Experimental Jaime Henao Jaramillo.

Las constantes ecofisiológicas resultaron sensiblemente apropiadas para evaluar la calidad del suelo y sensibles a los cambios por la época climática y variedad de café, sembrada en los suelos estudiados.

Agradecimientos. Al Centro de Ecología del Instituto Venezolano de Investigaciones Científicas (IVIC); al Laboratorio de Suelos de la Universidad de Córdoba (Colombia), por su colaboración en el análisis de algunos parámetros químicos del suelo. Financiación: Este estudio fue financiado por el Laboratorio de Ecología de Suelos del Instituto Venezolano de Investigaciones Científicas (IVIC). Conflictos de intereses: El manuscrito fue preparado y revisado con la participación de todos los autores, quienes declaran que no existe conflicto de intereses que ponga en riesgo la validez de los resultados presentados.

\section{REFERENCIAS}

1. ABERA, G.; WOLDE-MESKEL, E. 2013. Soil properties, and soil organic carbon stocks of tropical Andosol under different land uses. Open J. Soil Science. 3(3):153-162. https://doi.org/10.4236/ojss.2013.33018

2. ANDERSON, T.H.; DOMSCH, K.H. 1989. Ratios of microbial biomass carbon to total organic carbon in arable soils. Soil Biol. Biochem. 21:471-479. https://doi. org/10.1016/0038-0717(89)90117-X

3. ANDERSON, T.H.; DOMSCH, K.H. 1993.The metabolic quotient for $\mathrm{CO} 2$ (qCO2) as a specific activity parameter to assess the effects of environment conditions, such as $\mathrm{pH}$, on the microbial biomass of forest soils. Soil Biol. Biochem. 25(3):393-395. https://doi.org/10.1016/00380717(93)90140-7

4. ANDERSON, M.J.; GORLEY, R.N.; CLARKE, K.R. 2008. PERMANOVA + for PRIMER. Guide to software and statistical methods. Plymouth, U.K. PRIMER-E. 214p.

5. AZEVEDO JUNIOR, R.R.; SANTOS, J.B.; BARETTA, D.; RAMOS, A.C.; PEREIRA, A.P.; CARDOSO, E.J.B.N. 2017. Chemical y microbiological soil properties in organic and conventional management systems of Coffea arabica L. 
J. Plant Nutrition. 40:2076-2086. https://doi.org/10.1080 /01904167.2017.1346128

6. BALOTA, E.L.; CHAVES, J.C. 2011. Microbial activity in Soil cultivated with different summer legumes in Coffee Crop. Brazilian Archives of Biology and Technology. 54(1):35-44. https://doi.org/10.1590/S1516-89132011000100005

7. BASTIDA, F.; ZSOLNAY, A.; HERNÁNDEZ, T.; GARCÍA, C. 2008. Past, Present and Future of soil quality índices: A biological perspective. Geoderma. 147:159-171. https:// doi.org/10.1016/j.geoderma.2008.08.007

8. BECK, T.; ŐHLINGER, R.; BAUMGARTEN, A. 1995. Substrate-Induced Respiration. En: Schinner, F.; Öhlinger, R.; Kandeler, E.; Margesin, R. (eds.). Methods in Soil Biology. Springer Verlag (Berlin). p.64-68.

9. CARDOSO, I.M.; SOUZA, H.N.; MENDONCA, E.S. 2005. Biodiversidade, recurso genético e cuidados fitosanitários. R. Ação Ambiental. 31:18-20.

10. DILLY, O. 2005. Microbial Energetics in Soils. In: Varma, A.; Buscot, F. (eds.) Microorganisms in Soils: Roles in Genesis and Functions. Springer. Berlin Heidelberg. p.123-138. https://doi.org/10.1007/b137872

11. DURANGO, W.; URIBE, L.; HENRÍQUEZ, C.; MATA, R. 2015. Respiración, biomasa microbiana y actividad fosfatasa del suelo en dos agroecosistemas y un bosque en Turrialba, Costa Rica. Agronomía Costarricense. 39(1):37-46.

12. GLAESER, D.; MERCANTE, F.; ALVES, M.; DA SILVA, R.; KOMORI, O. 2010. Biomassa microbiana do solo sob sistemas de manejo orgánico em cultivos de café. Ensaios e Ciência: Ciências Biológicas, Agrárias e da Saúde. 14(2):103114.

13. HEANES, D.L. 1984. Determination of total organic-C in soils by an improved chromic acid digestion and spectrophotometric procedure. Communications in Soil Science and Plant Analysis. 15(10):1191-1213. https://doi. org/10.1080/00103628409367551

14. HERNÁNDEZ, T.; GARCIA, C. 2003. Estimación de la respiración microbiana del suelo. En: García, C.; Gil-Sotres, F.; Hernández, T.; Trasar-Cepeda, M. (eds.). Técnicas de Análisis de Parámetros Bioquímicos en suelos. Medida de Actividades Enzimáticas y Biomasa Microbiana. Editorial Mundi-Prensa. Madrid (España). p.322-324.

15. HÖPER, H. 2006. Substrate-induced respiration. In: Bloem, J., Hopkins, D.H., Benedetti, A. (Eds.), Microbiological Methods for Assessing Soil Quality. CABI Publishing, Wallingford, UK, p.84-92.
16. LAMMEL, D.R.; AZEVEDO, L.C.; PAULA, A.M.; ARMAS, R.D.; BARETTA, D.; CARDOSO, E.J. 2015. Microbiological and faunal soil attributes of coffee cultivation under different management systems in Brazil. Braz. J. Biol. 75(4):894-905. https://doi.org/10.1590/1519-6984.02414

17. MARTÍNEZ-SALGADO, M.; GUTIÉRREZ-ROMERO, V.; JANNSENS, M.; ORTEGA-BLU, R. 2010. Biological soil quality indicators: a review. In: Méndez-Vilas, A. (ed.). Current research, technology and education in applied microbiology and microbial biotechnology. Microbiology Series No 2. Formatex, Badajoz (España). p.319-328. https://doi.org/10.1016/j.enzmictec.2006.07.001

18. NOTARO, K.A.; MEDEIROS, E.V.; DUDA, G.P.; SILVA, A.O.; MOURA, P.A. 2014. Agroforestry systems, nutrients in litter and microbial activity in soils cultivated with coffee at high altitude. Scientia Agricola 71(2):87-95. https://doi. org/10.1590/s0103-90162014000200001

19. PABST, H.; KŸHNEL, A.; KUZYAKOV, Y. 2013. Effect of land-use and elevation on microbial biomass and water extractable carbon in soils of Mt. Kilimanjaro ecosystems. Applied Soil Ecology. 67(1):10-19. https://doi. org/10.1016/j.apsoil.2013.02.006

20. PAOLINI GÓMEZ, J.; ARELLANO, R.; GONZALEZ, L.; GUDIÑO, C. 2008. Mineralización del carbono en suelos cafetaleros del estado Trujillo (Venezuela). En: Blesa, M.; Alfonso, M.; Torres, R. (eds). Las Fronteras de la Física y Química Ambiental en Ibero América. UNSAM. Buenos Aires. p.174-180.

21. PAOLINI GÓMEZ, J.E. 2017. Actividad microbiológica y biomasa microbiana en suelos cafetaleros de los Andes venezolanos. Terra Latinoamericana. 36(1):13-22. https:// doi.org/10.28940/terra.v36i1.257

22. PELL, M.; STENSTRÖM, J.; GRANHALL, U. 2006. Soil respiration. In: Bloem, J. Hopking, D.W.; Benedetti, A. (eds.). Microbiological methods for assessing soil quality. $\mathrm{CAB}$ International, Wallingford, UK. p.117-126.

23. PERFECTO, I.; ARMBRECHT, I. 2002. The coffee agroecosystem in the Neotropics: combining ecological and economic goals. En: Vandermeer, J.H. (ed.) Tropical Agroecosystems. Imprint CRC Press p.159-194. https://doi. org/10.1201/9781420039887

24. RAPIDEL, B.; AKKINNE, C.; CERDAN, C.; MEYLAN, L.; FILHO, E.V.; AVELINO, J. 2015. Efectos ecológicos y productivos del asocio de árboles de sombra con café en sistemas agroforestales. En: Montagnini, F.; Somarriba, E.; Murgueitio, E.; Fassola, M.; Eibl, B. (eds.). Sistemas agroforestales: funciones productivas, socioeconómicas y ambientales. Serie técnica. Informe técnico 402. CATIE, 
Turrialba, Costa Rica. Editorial CIPAV, Cali (Colombia). p.5-19.

25. SADEGHIAN, S.; GONZÁLEZ, H. 2012. Alternativas generales de fertilización para cafetales en etapa de producción. Avances Técnicos Cenicafé No. 424. Gerencia Técnica. Programa de Investigación Científica. Fondo Nacional del Café. Manizales - Colombia. 8p.

26. SILVA-PARRA, A.; COLMENARES-PARRA, C.; ÁLVAREZ-ALARCÓN, J. 2017. Análisis multivariado de la fertilidad de los suelos en sistemas de café orgánico en Puente Abadia, Villavicencio. Rev. U.D.C.A Act. \& Div. Cient. 20(2):289-298. https://doi.org/10.31910/rudca.v20. n2.2017.387
27. SINGER, M.J.; MUNNS, D.N. 2006. Soils, an introduction. 6th Ediion. Pearson-Prentice Hall (Upper Saddle River, New Jersey). p.158-168.

28. SPARLING, G.P. 1992. Ratio between microbial biomass carbon to soil organic carbon as a sensitive indicator of changes in soil organic matter. Aust. J. Soil Research. 30(2):195-207. https://doi.org/10.1071/SR9920195

29. TORRES, S.; MADERO, L.; PERDOMO, C. 2009. Perfiles de suelos que integran la colección del centro de información y referencia de suelos (CIRS). (Características y propiedades más relevantes). Centro de Información y Referencia de Suelos (CIRS), Instituto de Edafología, Facultad de Agronomía UCV. Maracay. 217p. 